\title{
Malignant Phyllodes Tumor
}

National Cancer Institute

\section{Source}

National Cancer Institute. Malignant Phyllodes Tumor. NCI Thesaurus. Code C4275.

A phyllodes tumor with sarcomatous stroma. The sarcomatous component is usually of the fibrosarcomatous type. Liposarcomatous, chondrosarcomatous, osteosarcomatous, or rhabdomyosarcomatous differentiation may also occur in the stroma. It may recur and metastasize following surgical resection. The lung and skeleton are the anatomic sites most frequently involved by metastases. 